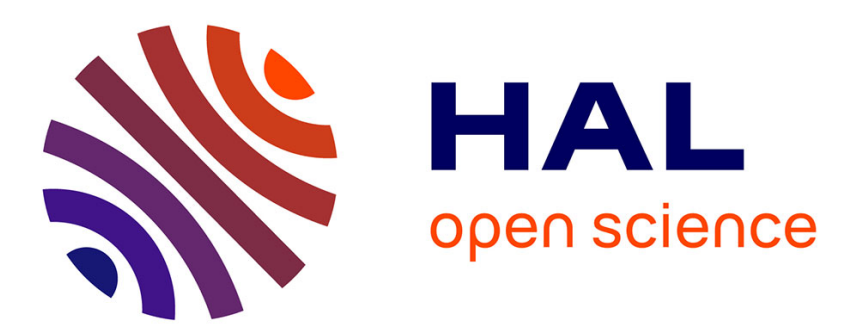

\title{
Dynamic modeling and design optimization of a 3-DOF spherical parallel manipulator
}

\author{
Guanglei Wu, Stéphane Caro, Shaoping Bai, Jørgen Kepler
}

\section{To cite this version:}

Guanglei Wu, Stéphane Caro, Shaoping Bai, Jørgen Kepler. Dynamic modeling and design optimization of a 3-DOF spherical parallel manipulator. Robotics and Autonomous Systems, 2014, 62, pp.1377 - 1386. 10.1016/j.robot.2014.06.006 . hal-01084693

\section{HAL Id: hal-01084693 https://hal.science/hal-01084693}

Submitted on 19 Nov 2014

HAL is a multi-disciplinary open access archive for the deposit and dissemination of scientific research documents, whether they are published or not. The documents may come from teaching and research institutions in France or abroad, or from public or private research centers.
L'archive ouverte pluridisciplinaire HAL, est destinée au dépôt et à la diffusion de documents scientifiques de niveau recherche, publiés ou non, émanant des établissements d'enseignement et de recherche français ou étrangers, des laboratoires publics ou privés. 


\title{
Dynamic Modeling and Design Optimization of a 3-DOF Spherical Parallel Manipulator
}

\author{
Guanglei $\mathbf{W u}^{1}$, Stéphane $\mathrm{Caro}^{2}$, Shaoping $\mathrm{Bai}^{1 *}$, Jørgen Kepler ${ }^{1}$ \\ ${ }^{1}$ Department of Mechanical and Manufacturing Engineering \\ Aalborg University, DK-9220 Aalborg, Denmark \\ E-mail: $\{g w u$, shb, jk\}@m-tech.aau.dk \\ ${ }^{2}$ CNRS, Institut de Recherche en Communications et Cybernétique de Nantes \\ UMR CNRS n 6597, 44321 Nantes, France \\ E-mail: Stephane.Caro@irccyn.ec-nantes.fr
}

\begin{abstract}
This paper deals with the dynamic modeling and design optimization of a three Degree-of-Freedom spherical parallel manipulator. Using the method of Lagrange multipliers, the equation of motion is derived by considering its motion characteristics, namely, all the components rotating about the center of rotation. Using the derived dynamic model, a multiobjective optimization problem is formulated to optimize the structural and geometric parameters of the spherical parallel manipulator. The proposed approach is illustrated with the design optimization of an unlimited-roll spherical parallel manipulator with a main objective to minimize the mechanism mass in order to enhance both kinematic and dynamic performances.
\end{abstract}

Keywords: Spherical parallel manipulator, dynamic modeling, multiobjective design optimization, Pareto-front, scatter matrix

\section{Introduction}

The design of three Degree-of-Freedom (3-DOF) spherical parallel manipulators (SPMs) can consider many criteria, such as workspace $[1,2,3]$, dexterity [4, 5, 6], dynamics [7], singularity avoidance [8], stiffness $[9,10]$. These evaluation criteria can be classified into two groups: one relates to the kinematic performance while the other relates to the kinetostatic/dynamic performance of the manipulator [11]. Most of the SPMs find their applications as orienting devices, such as camera orienting and medical instrument alignment $[12,13]$, therefore, the kinematic aspects, mainly, workspace and dexterity,

\footnotetext{
${ }^{*}$ Corresponding author, Tel: +45 9940 9291; Fax: +45 99407110.
} 
were extensively studied in the literature. On the other hand, the dynamics received less attention. Staicu [7] used the principle of virtual work to derive the inverse dynamics of the Agile Wrist [10], in which recursive matrix relations for kinematics and dynamics were established. When the SPMs are used to build active spherical manipulators, for instance, wrist joint [14], the dynamic characteristics is of importance in their design and applications.

This work develops a dynamic model with the classical approach of Lagrange multipliers, which takes all the mobile components into consideration to calculate the power consumption effectively. The equation of motion for the SPMs is modeled with the motion characteristics, namely, all the bodies rotating about a fixed point (center of rotation). The derived dynamic model can be used either to assess the dynamic performance or in the design optimization.

In general, a robot design process has to simultaneously deals with the kinematic and kinetostatic/dynamic aspects, both of which include a number of performance measures that essentially vary throughout the workspace. This can be effectively achieved by virtue of multiobjective optimization method. The multiobjective optimization problems of parallel manipulators (PMs) have been reported in the literature, where various approaches of multiobjective optimization have been applied to different types of PMs, while considering kinematic, dynamic and static criteria [11, 15, 16, 17, 18]. However, a systematic approach lacks in the optimum design for this class of SPMs, as the static/dynamic performance received relatively less attention as mentioned above.

This paper focuses on the dynamic modeling and design optimization of the SPMs. A dynamic model of the SPM is derived based on the Lagrange equations. Based on the dynamics, together with the kinematics and stiffness of the manipulator, a multiobjective design optimization method is proposed for SPMs, aiming to formulate a general approach for the SPMs in the early design stage. The multi-objective design optimization problem is applied to a 3-DOF unlimited-roll SPM, for which the Pareto-optimal solutions are obtained with a genetic algorithm.

\section{Architecture of SPMs}

A general spherical parallel manipulator is shown in Fig. 1. The $i$ th leg consists of three revolute joints, whose axes are parallel to the unit vectors $\mathbf{u}_{i}, \mathbf{v}_{i}$, and $\mathbf{w}_{i}$. All three legs have identical architectures, defined by angles $\alpha_{1}, \alpha_{2}, \beta$ and $\gamma$, where $\beta$ and $\gamma$ define the geometry of two regular pyramids of the base and mobile platforms. Both the two platforms are assumed rigid. The origin $O$ of the base coordinate system $\mathcal{F}_{a}$ is located at the center of rotation. The $z$ axis is normal to the bottom surface of the base pyramid and points upwards, while the $y$ axis is located in the plane spanned by the $z$ axis and $\mathbf{u}_{1}$ vector.

The SPM under study is a special case of $\gamma=0$ [3], where the pyramids of the base platform is degenerated to a line segment, as shown in Fig. 2(a). The axes of the three active revolute joints are coincident with the $z$ axis and it consists only of three curved links connected to its mobile platform. The links are driven by actuators moving independently on a circular guide via pinion and gear-ring transmissions, which can replace the serial chains based wrist mechanisms as displayed Fig. 2(b). 


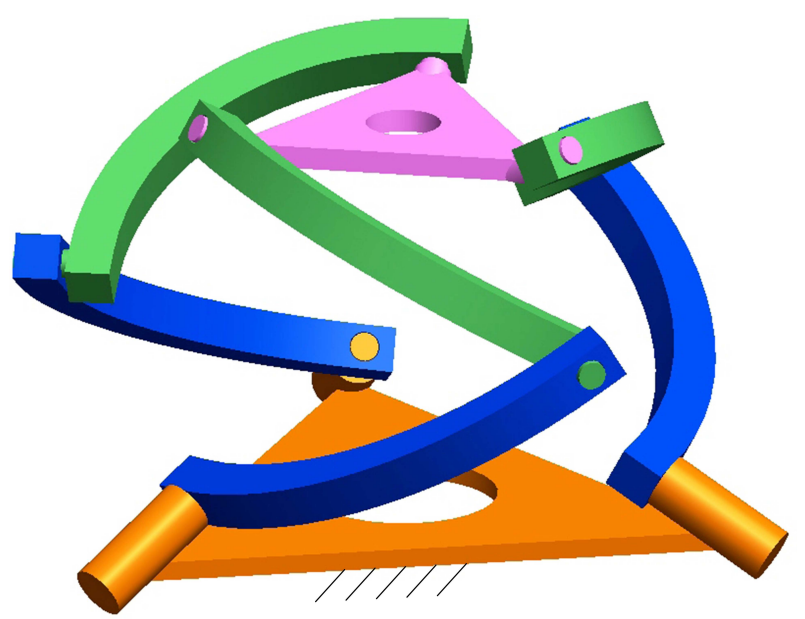

(a)

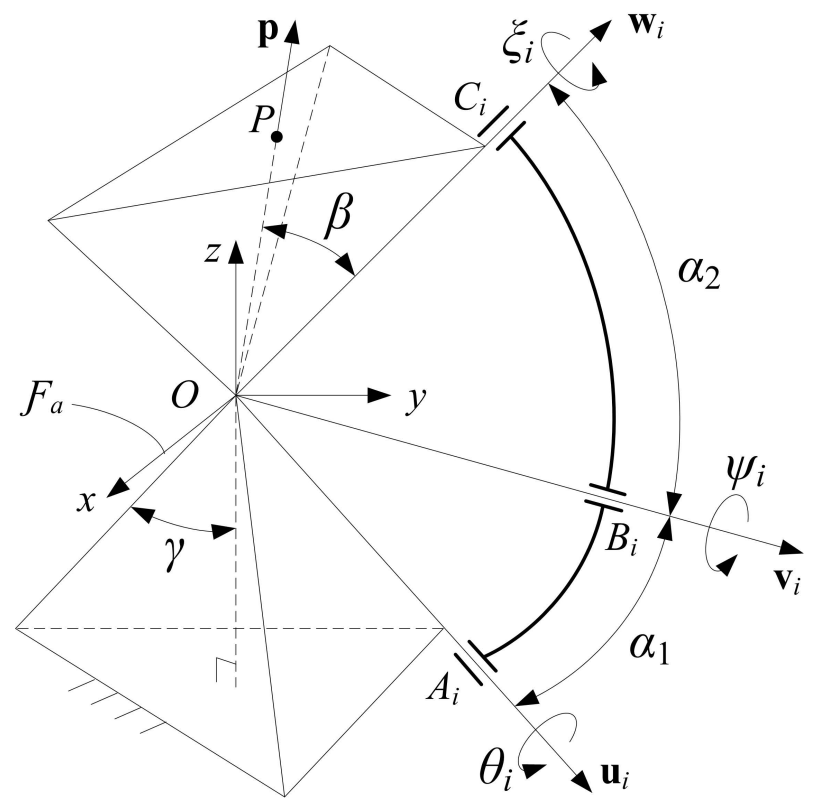

(b)

Figure 1: Architecture of a general SPM: (a) overview, (b) parameterization of the $i$ th leg.

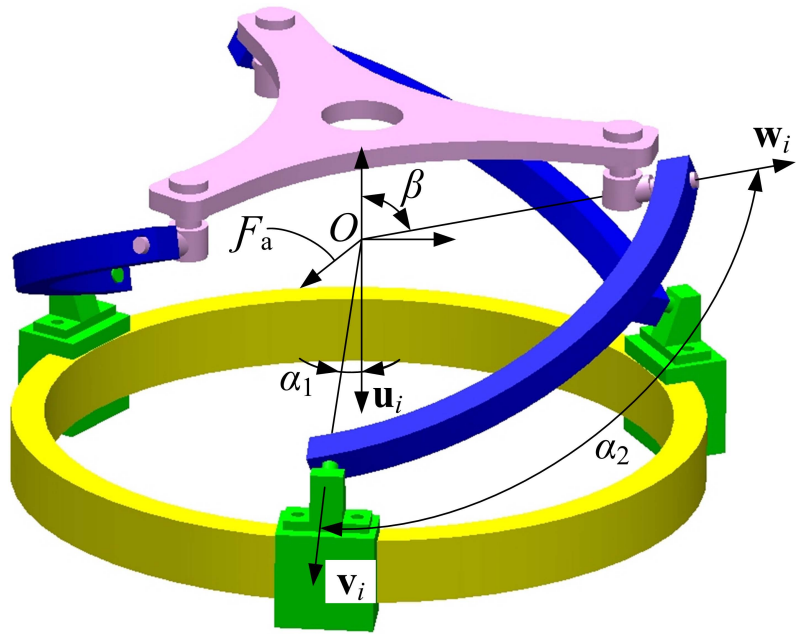

(a)

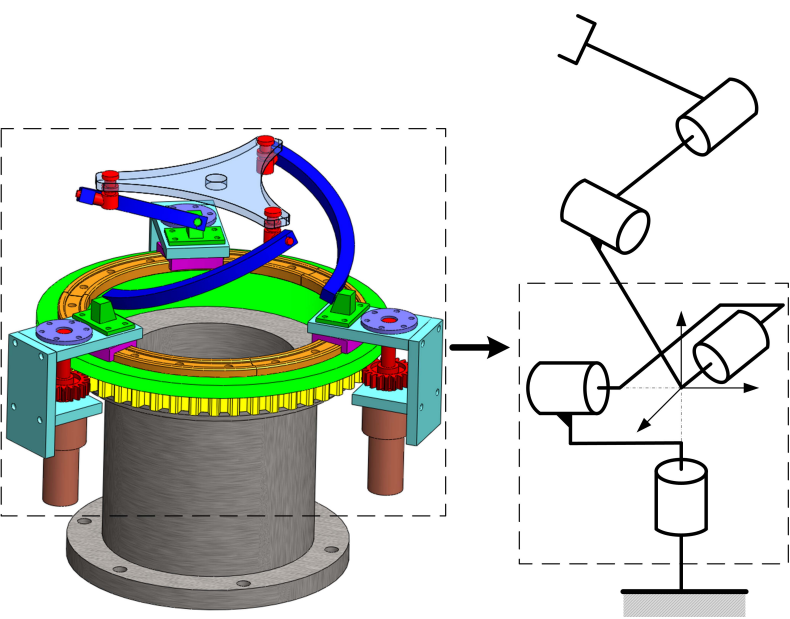

(b)

Figure 2: (a) 3-DOF unlimited-roll SPM, which is a special case of the general SPM with $\gamma=0$; (b) its application as spherically actuated joint. 


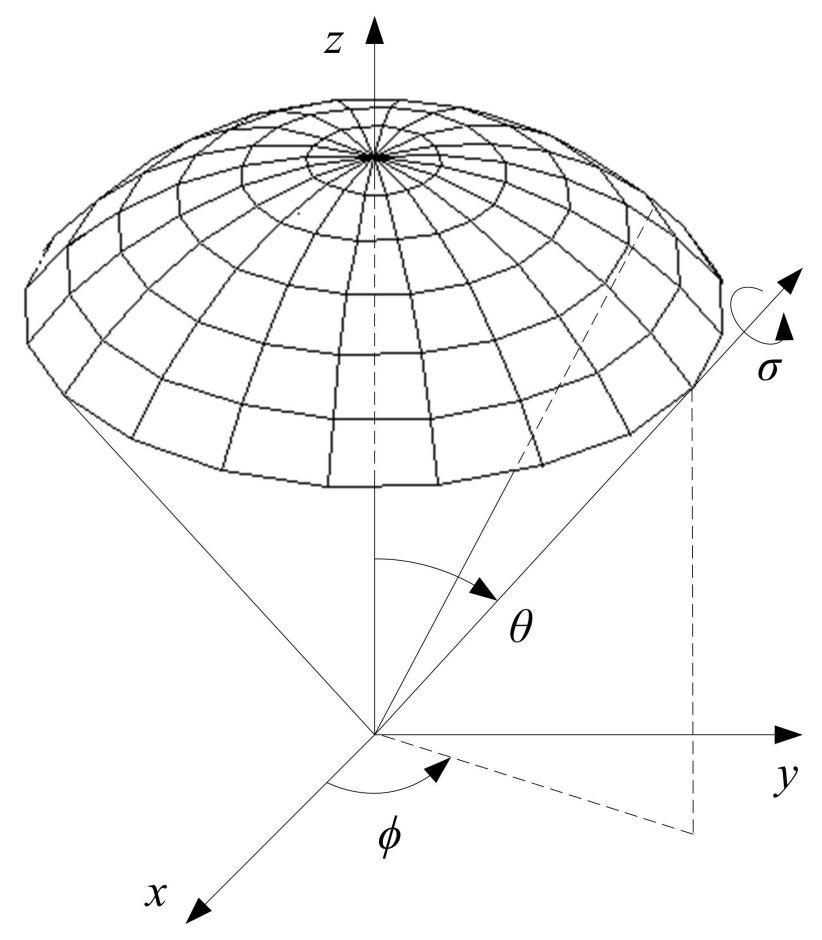

Figure 3: Orientation representation of the azimuth-tilt-torsion angles.

\section{Dynamic Modeling of SPMs}

The orientation of the mobile platform (MP) is described by the azimuth-tilt-torsion $(\phi-\theta-\sigma)$ angles [19] as displayed in Fig. 3, for which the rotation matrix is expressed as:

$$
\mathbf{Q}=\mathbf{R}_{z}(\phi) \mathbf{R}_{y}(\theta) \mathbf{R}_{z}(\sigma-\phi)
$$

where $\phi \in(-\pi, \pi], \theta \in[0, \pi), \sigma \in(-\pi, \pi]$.

Under the prescribed coordinate system, unit vector $\mathbf{u}_{i}$ is derived as

$$
\mathbf{u}_{i}=\left[\begin{array}{lll}
-\sin \eta_{i} \sin \gamma & \cos \eta_{i} \sin \gamma & -\cos \gamma
\end{array}\right]^{T}
$$

where $\eta_{i}=2(i-1) \pi / 3, i=1,2,3$.

Unit vector $\mathbf{v}_{i}$ of the axis of the intermediate revolute joint in the $i$ th leg is expressed as:

$$
\mathbf{v}_{i}=\left[\begin{array}{c}
-\mathrm{s} \eta_{i} \mathrm{~s} \gamma \mathrm{c} \alpha_{1}+\left(\mathrm{c} \eta_{i} \mathrm{~s} \theta_{i}-\mathrm{s} \eta_{i} \mathrm{c} \gamma \mathrm{c} \theta_{i}\right) \mathrm{s} \alpha_{1} \\
\mathrm{c} \eta_{i} \mathrm{~s} \gamma \mathrm{c} \alpha_{1}+\left(\mathrm{s} \eta_{i} \mathrm{~s} \theta_{i}+\mathrm{c} \eta_{i} \mathrm{c} \gamma \mathrm{c} \theta_{i}\right) \mathrm{s} \alpha_{1} \\
-\mathrm{c} \gamma \mathrm{c} \alpha_{1}+\mathrm{s} \gamma \mathrm{c} \theta_{i} \mathrm{~s} \alpha_{1}
\end{array}\right]
$$

Unit vector $\mathbf{w}_{i}$ of the top revolute joint in the $i$ th leg, is a function of the orientation of the mobile platform described as

$$
\mathbf{w}_{i}=\left[\begin{array}{lll}
w_{i x} & w_{i y} & w_{i z}
\end{array}\right]^{T}=\mathbf{Q w}_{i}^{*}
$$


where $\mathbf{w}_{i}^{*}$ is the unit vector for the axis of the top revolute joint of the $i$ th leg when the mobile platform (MP) reaches its home configuration, i.e., the MP orientation in the reference frame, which is given as

$$
\mathbf{w}_{i}^{*}=\left[\begin{array}{lll}
-\sin \eta_{i} \sin \beta & \cos \eta_{i} \sin \beta & \cos \beta
\end{array}\right]^{T}
$$

\subsection{Kinematic Jacobian matrix}

The relationship between the angular velocity of the mobile platform $\boldsymbol{\omega}=\left[\begin{array}{ll}\omega_{x} & \omega_{y} \omega_{z}\end{array}\right]^{T}$ and the input angle velocity $\dot{\boldsymbol{\theta}}=\left[\begin{array}{lll}\dot{\theta}_{1} & \dot{\theta}_{2} & \dot{\theta}_{3}\end{array}\right]^{T}$ is expressed as

$$
\mathbf{A} \omega=\mathbf{B} \dot{\theta}
$$

with

$$
\begin{aligned}
& \mathbf{A}=\left[\begin{array}{lll}
\mathbf{a}_{1} & \mathbf{a}_{2} & \mathbf{a}_{3}
\end{array}\right]^{T} ; \quad \mathbf{a}_{i}=\mathbf{v}_{i} \times \mathbf{w}_{i} \\
& \mathbf{B}=\operatorname{diag}\left[\begin{array}{lll}
b_{1} & b_{2} & b_{3}
\end{array}\right] ; \quad b_{i}=\left(\mathbf{u}_{i} \times \mathbf{v}_{i}\right) \cdot \mathbf{w}_{i}
\end{aligned}
$$

where matrices $\mathbf{A}$ and $\mathbf{B}$ are named the forward and inverse Jacobian matrices of the manipulator, respectively. The kinematic Jacobian matrix $\mathbf{J}$ of the manipulator [1] is obtained as

$$
\mathbf{J}=\mathbf{B}^{-1} \mathbf{A}=\left[\begin{array}{lll}
\mathbf{j}_{1} & \mathbf{j}_{2} & \mathbf{j}_{3}
\end{array}\right]^{T} ; \quad \mathbf{j}_{i}=\mathbf{a}_{i} / b_{i}
$$

\subsection{Inverse dynamic modeling}

The motions of the SPM bodies are shown in Fig. 4. The relationship between the angle rates $\dot{\varphi}=\left[\begin{array}{lll}\dot{\phi} & \dot{\theta} & \dot{\sigma}\end{array}\right]^{T}$ and the angular velocity $\boldsymbol{\omega}$ is found as $[20]$ :

$$
\left[\begin{array}{c}
\omega_{x} \\
\omega_{y} \\
\omega_{z}
\end{array}\right]=\left[\begin{array}{ccc}
-\mathrm{s} \theta \mathrm{c} \phi & -\mathrm{s} \phi & \mathrm{s} \theta \mathrm{c} \phi \\
-\mathrm{s} \theta \mathrm{s} \phi & \mathrm{c} \phi & \mathrm{s} \theta \mathrm{s} \phi \\
1-\mathrm{c} \theta & 0 & \mathrm{c} \theta
\end{array}\right]\left[\begin{array}{c}
\dot{\phi} \\
\dot{\theta} \\
\dot{\sigma}
\end{array}\right] \quad \text { or } \quad \boldsymbol{\omega}=\mathbf{\Phi} \dot{\boldsymbol{\varphi}}
$$

The dynamics of the SPM can be solved by using the Lagrange equations [21] below

$$
\frac{\mathrm{d}}{\mathrm{d} t}\left(\frac{\partial L}{\partial \dot{\mathbf{q}}}\right)-\frac{\partial L}{\partial \mathbf{q}}+\mathbf{C}_{q}^{T} \boldsymbol{\lambda}=\mathbf{Q}_{e x}
$$

where $L \equiv T-V$ is the Lagrangian of the system, including the mobile platform and the three legs, and $\mathbf{q}=\left[\theta_{1}, \theta_{2}, \theta_{3}, \phi, \theta, \sigma\right]^{T}$. Moreover, $\mathbf{Q}_{e x}=\left[\boldsymbol{\tau}^{T}, \mathbf{0}\right]^{T} \in \mathbb{R}^{6}$ is the vector of external forces and vector $\boldsymbol{\tau}=\left[\tau_{1}, \tau_{2}, \tau_{3}\right]^{T}$ characterizes the actuator torques. Matrix $\mathbf{C}_{q}$ is the system's constraint Jacobian, which can be found from the velocity equation (6), namely,

$$
\mathbf{B} \dot{\boldsymbol{\theta}}-\mathbf{A} \boldsymbol{\omega}=\left[\begin{array}{ll}
\mathbf{B} & -\mathbf{A} \boldsymbol{\Phi}
\end{array}\right]\left[\begin{array}{ll}
\dot{\boldsymbol{\theta}}^{T} & \dot{\varphi}^{T}
\end{array}\right]^{T}=\mathbf{0}
$$




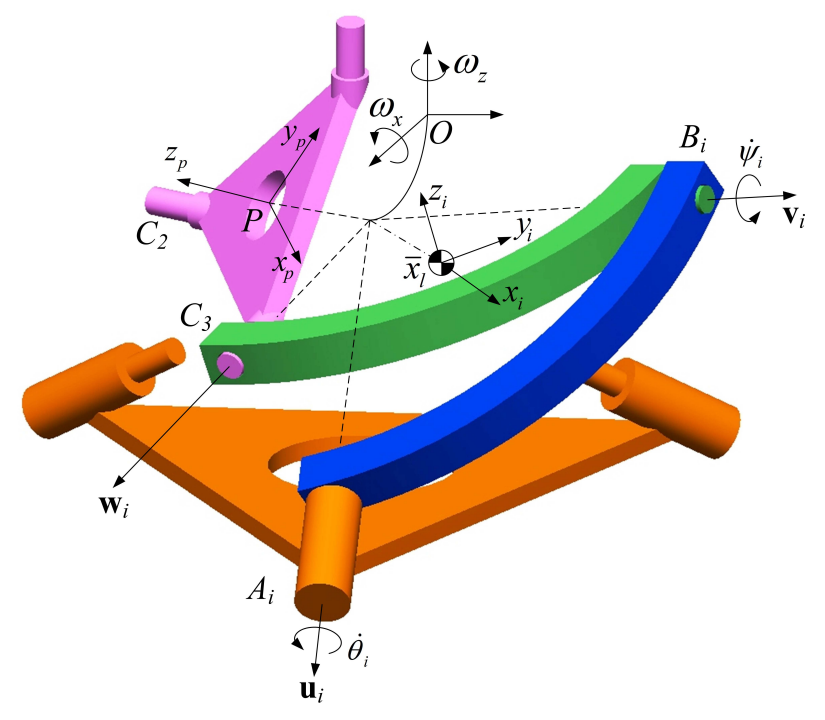

Figure 4: The movements of the mobile platform and a single leg.

therefore, the matrix of constraints is found as $\mathbf{C}_{q}=[\mathbf{B}-\mathbf{A} \mathbf{\Phi}]$. Moreover, $\boldsymbol{\lambda}=\left[\lambda_{1}, \lambda_{2}, \lambda_{3}\right]^{T}$ is a vector of Lagrange multipliers.

\subsubsection{Lagrangian of the mobile platform}

The local frame $\left(x_{p}, y_{p}, z_{p}\right)$ attached to the MP is established with the origin located at point $P$, i.e., the center of mass of the MP. Henceforth, the Lagrangian of the mobile platform is obtained as

$$
L_{p}=T_{p}-V_{p}=\frac{1}{2} \boldsymbol{\omega}^{T} \mathbf{I}_{p} \boldsymbol{\omega}-m_{p} R \cos \beta \mathbf{g}^{T} \mathbf{p}
$$

where $\mathbf{I}_{p}$ denotes the global inertia tensor of the mobile platform, which can be found in Appendix A. Moreover, $\mathbf{g}=[0,0,9.81]^{T}$.

\subsubsection{Lagrangian of a single leg}

The velocity $\dot{\psi}$ of the intermediate joint in $i$ th leg is found using the following equation

$$
\boldsymbol{\omega}=\dot{\theta}_{i} \mathbf{u}_{i}+\dot{\psi}_{i} \mathbf{v}_{i}+\dot{\xi}_{i} \mathbf{w}_{i}
$$

To eliminate $\dot{\theta}_{i}$ and $\dot{\xi}_{i}$, dot-multiplying Eqn. (13) on both sides with $\mathbf{u}_{i} \times \mathbf{w}_{i}$ yields

$$
\left(\mathbf{u}_{i} \times \mathbf{w}_{i}\right) \cdot \boldsymbol{\omega}=\dot{\psi}\left(\mathbf{u}_{i} \times \mathbf{w}_{i}\right) \cdot \mathbf{v}_{i} \text { or } \quad \dot{\psi}=\mathbf{j}_{\psi i}^{T} \boldsymbol{\omega}=\frac{\left(\mathbf{u}_{i} \times \mathbf{w}_{i}\right)^{T}}{\left(\mathbf{u}_{i} \times \mathbf{w}_{i}\right) \cdot \mathbf{v}_{i}} \boldsymbol{\omega}
$$

The angular velocity of the distal link in the $i$ th leg in the reference frame $(x, y, z)$ is found as $\varpi_{i}=\dot{\theta}_{i} \mathbf{u}_{i}+\dot{\psi}_{i} \mathbf{v}_{i}$. Let $\varpi_{l i}$ denote the corresponding angular velocity in the local frame $\left(x_{i}, y_{i}, z_{i}\right)$, 
we have

$$
\varpi_{i}=\left[\begin{array}{lll}
\mathbf{e}_{i x} & \mathbf{e}_{i y} & \mathbf{e}_{i z}
\end{array}\right] \varpi_{l i} \quad \text { or } \quad \varpi_{i}=\mathbf{E}_{i} \varpi_{l i}
$$

with

$$
\mathbf{e}_{i x}=\frac{\mathbf{v}_{i}+\mathbf{w}_{i}}{\left\|\mathbf{v}_{i}+\mathbf{w}_{i}\right\|}, \mathbf{e}_{i y}=\frac{\mathbf{v}_{i}-\mathbf{w}_{i}}{\left\|\mathbf{v}_{i}-\mathbf{w}_{i}\right\|}, \mathbf{e}_{i z}=\frac{\mathbf{v}_{i} \times \mathbf{w}_{i}}{\left\|\mathbf{v}_{i} \times \mathbf{w}_{i}\right\|}
$$

From Eqn. (15), we have $\varpi_{l i}=\mathbf{E}_{i}^{T} \varpi_{i}$. The Lagrangian of the $i$ th leg is derived as below

$$
L_{i}=T_{i}-V_{i}=\frac{1}{2} I_{l 1} \dot{\theta}_{i}^{2}+\frac{1}{2} \varpi_{i}^{T} \mathbf{I}_{l 2} \varpi_{i}-m_{l 1} \bar{x}_{1} \mathbf{g}^{T} \mathbf{h}_{i}-m_{l 2} \bar{x}_{2} \mathbf{g}^{T} \mathbf{e}_{i x}
$$

where $I_{l 1}$ is the proximal link's mass moment of inertia about $\mathbf{u}_{i}$, and $\mathbf{I}_{l 2}$ is the distal link's mass moment of inertia about point $O$. Moreover, $\bar{x}_{1}$ and $\bar{x}_{2}$ indicate the centers of the mass of the proximal and distal links, respectively, and $\mathbf{h}_{i}=\left(\mathbf{u}_{i}+\mathbf{v}_{i}\right) /\left\|\mathbf{u}_{i}+\mathbf{v}_{i}\right\|$. The details for a curved link can be found in Appendix A.

Substituting the Lagrangian $L_{p}$ and $L_{i}, i=1,2,3$, into Eqn. (10), the terms in the equation of motion for this dynamic system [21] can be derived. With an external moment vector $\mathbf{m}$, the actuator torques are expressed as:

$$
\boldsymbol{\tau}_{a}=\boldsymbol{\tau}-\mathbf{J}^{-T} \mathbf{m}
$$

This developed dynamic model can effectively compute the active forces/torques as it takes into account all the mobile components and external forces/moments. Compared to Staicu's work [7], the model developed in this work has a more compact form, which takes advantages of the unique feature of SPMs that involves only rotations. Such a formulation can be easily understood and implemented.

\section{Optimization Problem of the SPMs Design}

The foregoing derived dynamic model can be applied in the optimization procedure to obtain a design with optimal dynamic performance. Henceforth, this section formulates a design optimization problem for the SPMs based on their dynamic modeling. Besides, the kinematic and elastic performances are also employed to evaluate the SPM design. A predefined workspace is specified as a minimum pointing cone of $90^{\circ}$ opening with $360^{\circ}$ full rotation, i.e., $\theta \in\left[0, \theta_{\min }\right], \theta_{\min } \geq 45^{\circ},\{\phi, \sigma\} \in\left(-180^{\circ}, 180^{\circ}\right]$.

\subsection{Design variables}

Variables $\alpha_{1}, \alpha_{2}$ and $\beta$ are part of the geometric parameters of the SPM under study. Moreover, the radius $R$ of the link midcurve and the side length $a$ of a square cross section of the uniform curved links are included as design parameters as well. This implies that the curved link does not include details such as slots that may affect the total mass and structural strength [22]. As a consequence, the design variables of the optimization problem at hand are:

$$
\mathbf{x}=\left[\alpha_{1}, \alpha_{2}, \beta, a, R\right]
$$




\subsection{Objective functions}

The mechanism mass influences the dynamic performance, such as inertia, acceleration, etc., hence, minimizing the mass of moving bodies is one important consideration. The mass $m_{s p m}$ of the SPM includes the mass $m_{p}$ of the platform, the mass $m_{l}$ of the distal links, and the mass $m_{s}$ of the sliding units (or proximal links). The mass of the revolute joints is not considered for simplification, thus, the mass function is given as:

$$
m_{\text {spm }}=m_{p}+3 m_{l}+3 m_{s}
$$

As a result, the first objective function of the optimization problem is written as:

$$
f_{1}(\mathbf{x})=m_{\text {spm }} \quad \rightarrow \quad \min
$$

The dexterity of SPMs is another major concern in the manipulator design. A commonly used criterion to evaluate this kinematic performance is the global conditioning index (GCI) [4], which describes the isotropy of the kinematic performance. The GCI is defined over a workspace $\Omega$, which is calculated through a discrete approach in practice, namely,

$$
\mathrm{GCI}=\frac{\int_{\Omega} \kappa^{-1}(\mathbf{J}) \mathrm{d} W}{\int_{\Omega} \mathrm{d} W} \quad \text { or } \quad \mathrm{GCI}=\frac{1}{n} \sum_{k=1}^{n} \frac{1}{\kappa_{k}(\mathbf{J})}
$$

where $\kappa(\mathbf{J})$ is the condition number of the kinematic Jacobian matrix (8) and $n=n_{1} n_{2} n_{3}$ is the number of the workspace points, $n_{1}, n_{2}, n_{3}$ being the numbers of discrete points along $\phi, \theta, \sigma$, respectively. It is known that the higher the GCI, the better the performance. Hereby, the second objective function of the optimization problem is written as:

$$
f_{2}(\mathbf{x})=1-\mathrm{GCI}=\mathrm{MGCI} \rightarrow \min
$$

Henceforth, a modified global conditioning index (MGCI) is introduced for the purpose of optimization.

\subsection{Optimization constraints}

In this section, the kinematic constraints, condition number of the kinematic Jacobian matrix, elastic and dynamic performances of the manipulator are considered. Constraining the condition number of the Jacobian matrix aims to obtain a dexterous workspace free of singularity. Moreover, the constraints on the link strength and the actuator torque are also considered.

\subsubsection{Geometric constraints}

According to the determination of the design space reported in [3], the bounds of the parameters $\alpha_{1}$, $\alpha_{2}$ and $\beta$ subject to the prescribed workspace are stated as:

$$
45^{\mathrm{o}} \leq \alpha_{1} \leq 135^{\circ}, 45^{\mathrm{o}} \leq \alpha_{2} \leq 135^{\circ}, 45^{\circ} \leq \beta \leq 90^{\circ}
$$



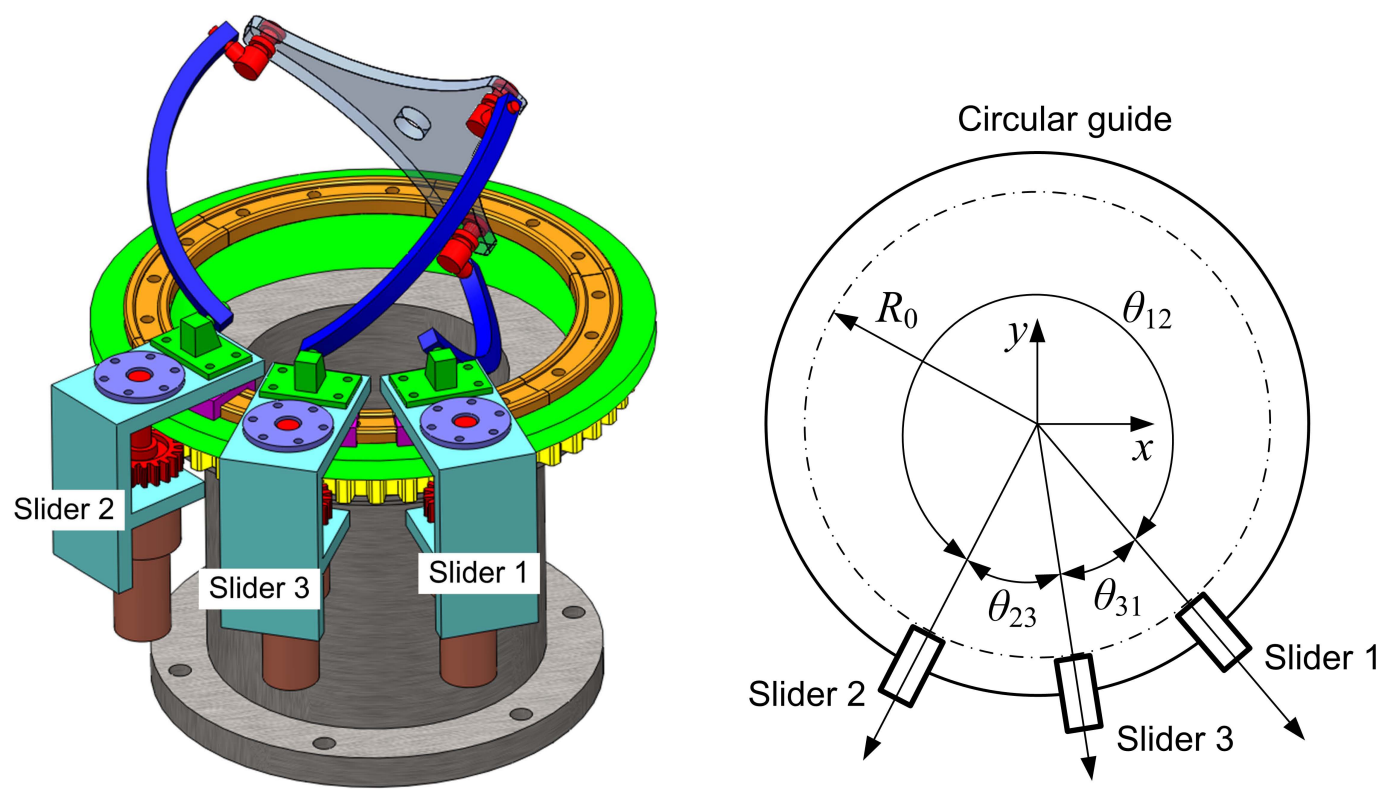

Figure 5: One extreme configuration of the sliding units.

In accordance with Fig. 5, the following constraints should be satisfied to avoid any collision,

$$
\begin{aligned}
& \theta_{i j} \geq \epsilon_{\theta}, \forall \theta_{i j} \in\left\{\theta_{12}, \theta_{23}, \theta_{31}\right\} \\
& R \sin \alpha_{1} \geq R_{0}
\end{aligned}
$$

where $\epsilon_{\theta}=10^{\circ}$ and $R_{0}=0.120 \mathrm{~m}$ are geometric parameters relative to the size of curved links.

\subsubsection{Condition number of the kinematic Jacobian matrix}

Minimizing MGCI, i.e., maximizing GCI, cannot prevent the prescribed workspace away from illconditioned configurations. For the design optimization to achieve a dexterous workspace, the minimum of the inverse condition number of the kinematic Jacobian matrix $\kappa^{-1}(\mathbf{J})$, based on the 2-norm, should be higher than a prescribed value throughout the workspace, say 0.1 , namely,

$$
\min \left(\kappa^{-1}(\mathbf{J})\right) \geq 0.1
$$

\subsubsection{Strength constraints}

The strength constraints are to ensure the SPM to produce allowable maximum point-displacement of the rotation center and angular deflection of the mobile platform subject to a given wrench. The deflections are computed by

$$
\Delta \mathbf{x}=\mathbf{K}^{-1} \mathbf{w} ; \Delta \mathbf{x}=\left[\begin{array}{ll}
\Delta \mathbf{p}^{T} & \Delta \varphi^{T}
\end{array}\right]^{T}, \mathbf{w}=\left[\begin{array}{ll}
\mathbf{0} & \mathbf{m}^{T}
\end{array}\right]^{T}
$$


where $\Delta \mathbf{p}=[\Delta x, \Delta y, \Delta z]^{T}$ and $\Delta \varphi=\left[\Delta \varphi_{x}, \Delta \varphi_{y}, \Delta \varphi_{z}\right]^{T}$ are the translational and rotational displacements, respectively, and $\mathbf{K}$ is the Cartesian stiffness matrix given in Appendix B. Let the static torque of the SPM within the range $\mathbf{m}=\left[ \pm m_{x, \max }, \pm m_{y, \max }, \pm m_{z, \max }\right]$, the strength constraints can be written as:

$$
\begin{aligned}
& -\epsilon_{p} \leq \Delta t \leq \epsilon_{p}, \forall \Delta t \in\left\{\Delta x_{k}, \Delta y_{k}, \Delta z_{k}\right\} \\
& -\epsilon_{r} \leq \Delta r \leq \epsilon_{r}, \forall \Delta r \in\left\{\Delta \varphi_{x, k}, \Delta \varphi_{y, k}, \Delta \varphi_{z, k}\right\}
\end{aligned}
$$

where $\epsilon_{p}$ and $\epsilon_{r}$ are acceptable translational and rotational errors, respectively, and $k=1, \ldots, n$ is the number of the discrete points defined in Eqn. (22).

It is noted that the model in Eqn. (27) includes only the compliances of curved links. The base and MP are considered rigid. Moreover, the joint compliance is not addressed in this paper, as revolute joints are not easily characterized by a generic representative stiffness due to the nonlinear kinematic joint stiffness upon the specific design. Such an issue could be well addressed through stiffness modeling researches.

\subsubsection{Actuation torque constraints}

With an external moment $\mathbf{m}=\left[m_{x}, m_{y}, m_{z}\right]^{T}$ applied on the MP, in accordance to Eqn. (7(b)), the actuator torques are redefined as: $\boldsymbol{\tau}_{a}=|\boldsymbol{\tau}|+\left|\mathbf{J}^{-T} \mathbf{m}\right|$. At any time, the components of the actuator torque vector $\tau_{a}$ should be smaller than the maximum continuous torque $T_{\max }$ of each actuator. As a result, the actuation torque constraints can be written as:

$$
\max \left\{\tau_{a}\right\} \leq T_{\max }
$$

Henceforth, two alternative trajectories describing the MP orientation:

$$
\begin{aligned}
& \text { (1) } \phi(t)=\pi \cos t, \theta(t)=\frac{\pi}{4}, \sigma(t)=\frac{\pi}{2} \cos t \\
& \text { (2) } \phi(t)=\frac{\pi}{2} \cos t, \theta(t)=\frac{\pi}{4}, \sigma(t)=\pi \cos t
\end{aligned}
$$

are integrated into the optimization procedure implemented with the Matlab/simulink package. The corresponding angular velocity and acceleration profiles of the mobile platform are shown in Fig. 6. 


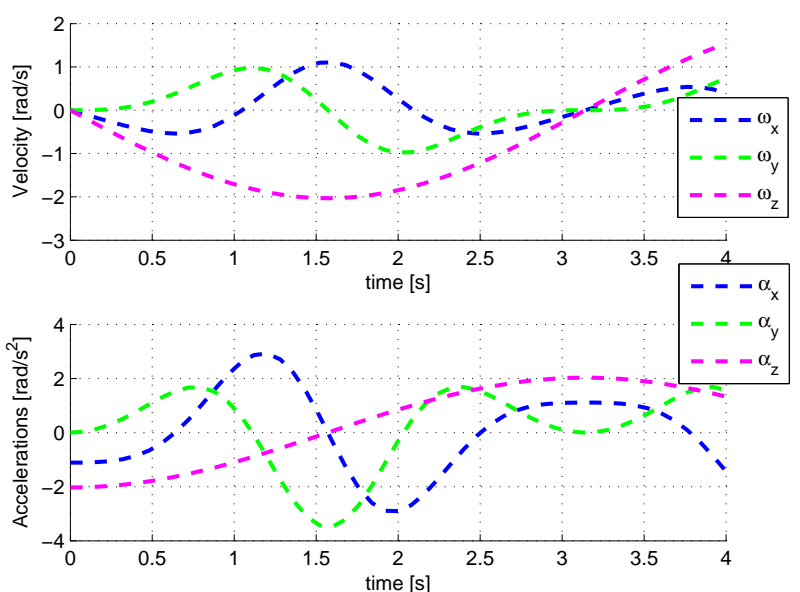

(a)

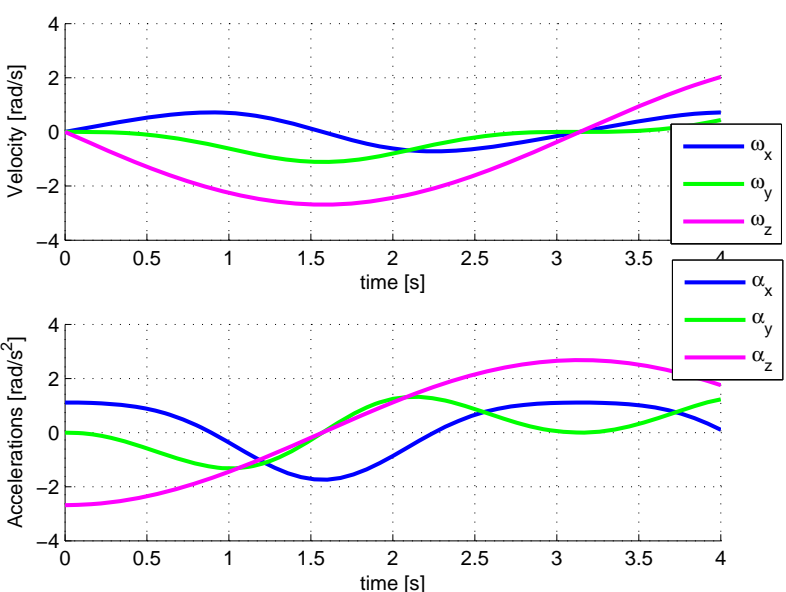

(b)

Figure 6: The angular velocity and acceleration profiles of the mobile platform: (a) trajectory 1; (b) trajectory 2 .

\subsection{Formulation of the multi-objective design optimization problem}

The multi-objective design optimization problem for the SPM is formulated as:

$$
\begin{aligned}
\text { minimize } & f_{1}(\mathbf{x})=m_{\text {spm }} \\
\text { minimize } & f_{2}(\mathbf{x})=M G C I \\
\text { over } & \mathbf{x}=\left[\alpha_{1} ; \alpha_{2} ; \beta ; a ; R\right] \\
\text { subject to } & g_{1}: R \sin \alpha_{1} \geq R_{0} \\
& g_{2}: \theta_{i j} \geq \epsilon_{\theta}, \forall \theta_{i j} \in\left\{\theta_{12}, \theta_{23}, \theta_{31}\right\} \\
& g_{3}: \theta_{\min } \geq 45^{\circ} \\
& g_{4}: \min \left(\kappa^{-1}(\mathbf{J})\right) \geq 0.1 \\
& g_{5}:-\epsilon_{p} \leq \Delta t \leq \epsilon_{p}, \forall \Delta t \in\left\{\Delta x_{k}, \Delta y_{k}, \Delta z_{k}\right\} \\
& g_{6}:-\epsilon_{r} \leq \Delta r \leq \epsilon_{r}, \forall \Delta r \in\left\{\Delta \varphi_{x, k}, \Delta \varphi_{y, k}, \Delta \varphi_{z, k}\right\} \\
& g_{7}: \max \left\{\boldsymbol{\tau}_{a}\right\} \leq T_{\max }
\end{aligned}
$$

\section{$5 \quad$ Results and Discussion}

\subsection{Validation of Dynamic Model}

Dynamic simulations were conducted with the developed SPM dynamic model, the obtained results being compared with those obtained with MSC Adams, utilizing the properties and simulation conditions given in Tables 1 and 2. The corresponding simulation results from the developed model and the Adams simulation are shown in Fig. 7, which shows a good agreement with each other. 
Table 1: Parameters of the SPM and the initial simulation condition.

\begin{tabular}{cccccc}
\hline \hline$\alpha_{1}[\mathrm{deg}]$ & $\alpha_{2}[\mathrm{deg}]$ & $\beta[\mathrm{deg}]$ & {$[\phi, \theta, \sigma][\mathrm{rad}]$} & {$\left[\dot{\theta}_{1}, \dot{\theta}_{2}, \dot{\theta}_{3}\right][\mathrm{rad} / \mathrm{s}]$} & $\mathbf{m}[\mathrm{Nm}]$ \\
\hline 60 & 75 & 75 & {$[0, \pi / 6,0]$} & {$[-6,-5,-7]$} & {$[0.1,0.1,0.1]$} \\
\hline \hline
\end{tabular}

Table 2: Mass and inertia properties of the SPM model.

\begin{tabular}{|c|c|c|c|c|}
\hline \multicolumn{2}{|c|}{ Mobile platform } & \multicolumn{2}{|c|}{ Curved link } & Sliding unit \\
\hline$m_{p}[\mathrm{~kg}]$ & $\mathbf{I}_{p}\left[10^{-4} \mathrm{~kg} \mathrm{~m}^{2}\right]$ & $m_{l}[\mathrm{~kg}]$ & $\mathbf{I}_{l}\left[10^{-4} \mathrm{~kg} \mathrm{~m}^{2}\right]$ & $m_{s}[\mathrm{~kg}]$ \\
\hline 0.332 & {$\left[\begin{array}{llll}3.855 & 3.855 & 7.688\end{array}\right]$} & 0.107 & {$\left[\begin{array}{llll}1.816 & 0.081 & 1.894\end{array}\right]$} & 0.123 \\
\hline
\end{tabular}
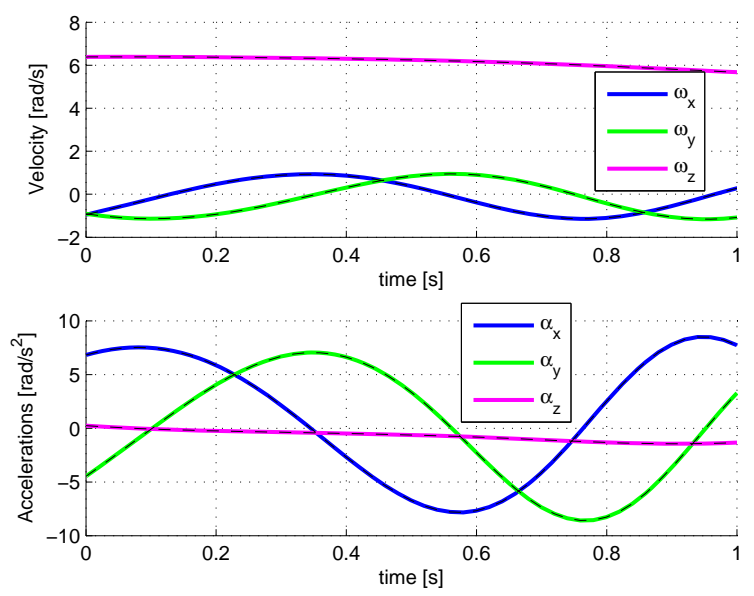

(a)

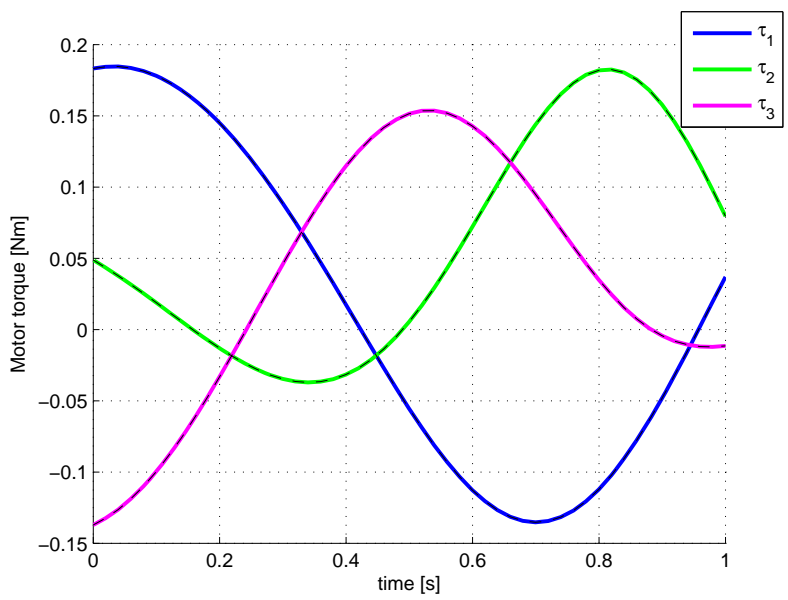

(b)

Figure 7: Simulation results (solid line stands for Matlab solver, dashed line for Adams): (a) motion of the mobile platform; (b) actuator torques. 
Table 3: Algorithm parameters of the implemented NSGA-II.

\begin{tabular}{ccccc}
\hline \hline $\begin{array}{c}\text { Population } \\
\text { size }\end{array}$ & $\begin{array}{c}\text { Number of } \\
\text { generations }\end{array}$ & $\begin{array}{c}\text { Directional crossover } \\
\text { probability }\end{array}$ & $\begin{array}{c}\text { Crossover } \\
\text { probability }\end{array}$ & $\begin{array}{c}\text { Distribution } \\
\text { index }\end{array}$ \\
\hline 40 & 200 & 0.5 & 0.9 & 20 \\
\hline \hline
\end{tabular}

Table 4: The lower and upper bounds of the design variables.

\begin{tabular}{cccccc}
\hline \hline & $\alpha_{1}[\mathrm{deg}]$ & $\alpha_{2}[\mathrm{deg}]$ & $\beta[\mathrm{deg}]$ & $a[\mathrm{~m}]$ & $R[\mathrm{~m}]$ \\
\hline $\mathbf{x}_{l b}$ & 45 & 45 & 45 & 0.005 & 0.120 \\
$\mathbf{x}_{u b}$ & 135 & 135 & 90 & 0.030 & 0.300 \\
\hline \hline
\end{tabular}

\subsection{Design Optimization}

The optimization procedure is applied to the SPM shown in Fig. 2(b). The actuation transmission mechanism is a combination of RE $35 \mathrm{~GB}$ actuator and GP $42 \mathrm{C}$ gearhead from Maxon [23]. The components are supposed to be made up of steel, and the mobile platform is supposed to be a regular triangle. The total mass $m_{s}$ of each slide unit, including the mass of the actuator, gearhead, pinions and the manufactured components, is equal to $m_{s}=2.1 \mathrm{~kg}$. Henceforth, the actuation stiffness is $K_{\text {act }}^{i}=$ $10^{6} \mathrm{~N} \cdot \mathrm{m} / \mathrm{rad}$ and the range of the static moment in Eqn. (27) is $\mathbf{m}=[ \pm 10, \pm 10, \pm 10] \mathrm{N} \cdot \mathrm{m}$, while the acceptable translational and rotational errors are $\epsilon_{p}=1 \mathrm{~mm}$ and $\epsilon_{r}=0.0349 \mathrm{rad}$, respectively. Moreover, the maximum continuous torque of the actuator is $T_{\max }=15 \mathrm{Nm}$ and the external moment applied on the mobile platform and expressed in the base frame along the trajectories defined by Eqn. (30) is $\mathbf{m}=[5,5,5]^{T} \mathrm{Nm}$.

The solutions of the previous optimization problem are non-dominated solutions, also called Paretooptimal solutions, which stand for solutions for which the corresponding objectives cannot be further improved without degrading others. Problem (31) is solved by the genetic algorithm NSGA-II [24] implemented in Matlab, for which the algorithm parameters are given in Table. 3. The lower and upper bounds of the design variables are shown in Table 4, denoted by $\mathbf{x}_{l b}$ and $\mathbf{x}_{u b}$, respectively.

The Pareto-front of the optimization problem at hand is shown in Fig. 8. Three Pareto-optimal solutions named ID-I, ID-II and ID-III on the Pareto-fronts, i.e., two extreme solutions and one intermediate solution, described in Table 5, are selected for further consideration. The CAD designs of these three Pareto-optimal solutions are shown in Fig. 9 and the corresponding dynamic simulations are illustrated in Fig. 10, respectively, from which it is seen that from ID-I to III, the maximum actuating torque increases. By comparison among the three groups of design variables in Table 5 , smaller $\alpha_{1}$ and larger $\alpha_{2}$ yield higher wrench capability under given input torques. Although the design ID-III has the lowest mass, it has the highest requirements on the actuators. From the kinematic and dynamic considerations, design ID-I can be selected for further application as an active joint.

Figure 11, obtained with plotmatrix and corrcoef functions in Matlab, illustrates the variational 


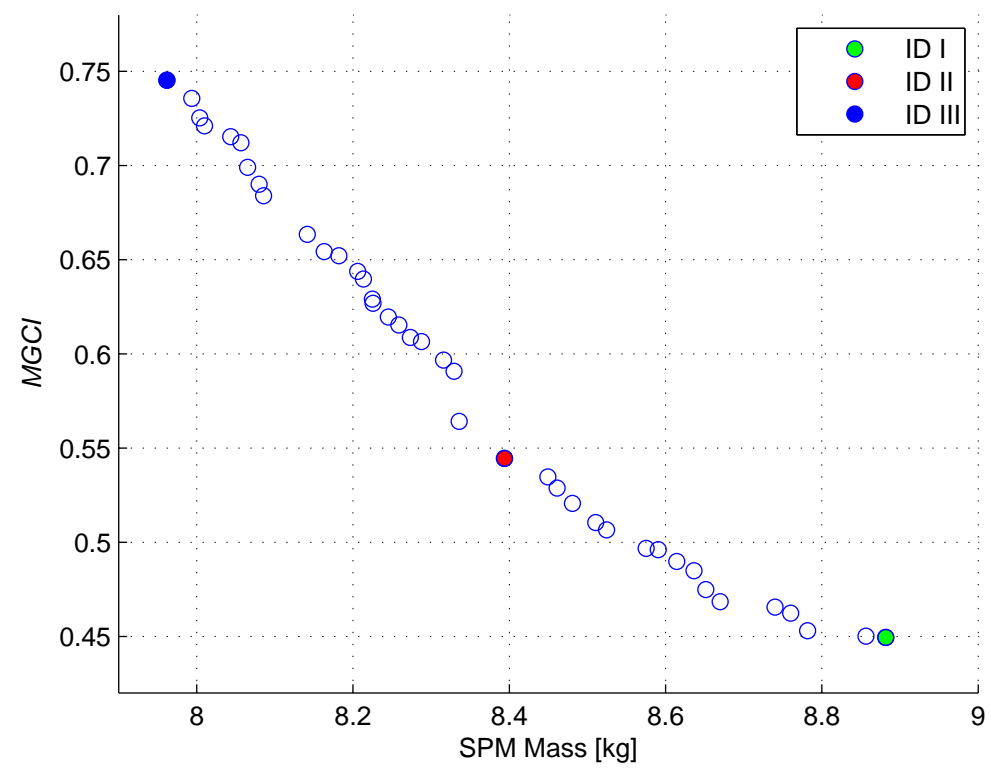

Figure 8: The Pareto-front of the multiobjective optimization problem (31).

Table 5: Three Pareto-optimal solutions.

\begin{tabular}{|c|c|c|c|c|c|c|c|c|}
\hline \multirow{2}{*}{$\begin{array}{c}\text { Design } \\
\text { ID }\end{array}$} & \multicolumn{5}{|c|}{ Variables } & \multicolumn{2}{|c|}{ Objectives } & \multirow[b]{2}{*}{$\min \left(\kappa^{-1}(\mathbf{J})\right)$} \\
\hline & $\alpha_{1}[\mathrm{deg}]$ & $\alpha_{2}[\mathrm{deg}]$ & $\beta$ [deg] & $a[\mathrm{~m}]$ & $R[\mathrm{~m}]$ & $m_{\text {spm }}[\mathrm{kg}]$ & $M G C I$ & \\
\hline I & 47.2 & 91.7 & 88.4 & 0.0120 & 0.1659 & 8.882 & 0.449 & 0.253 \\
\hline II & 51.9 & 85.0 & 88.3 & 0.0113 & 0.1525 & 8.394 & 0.545 & 0.240 \\
\hline III & 63.5 & 72.2 & 89.6 & 0.0119 & 0.1342 & 7.962 & 0.745 & 0.102 \\
\hline
\end{tabular}

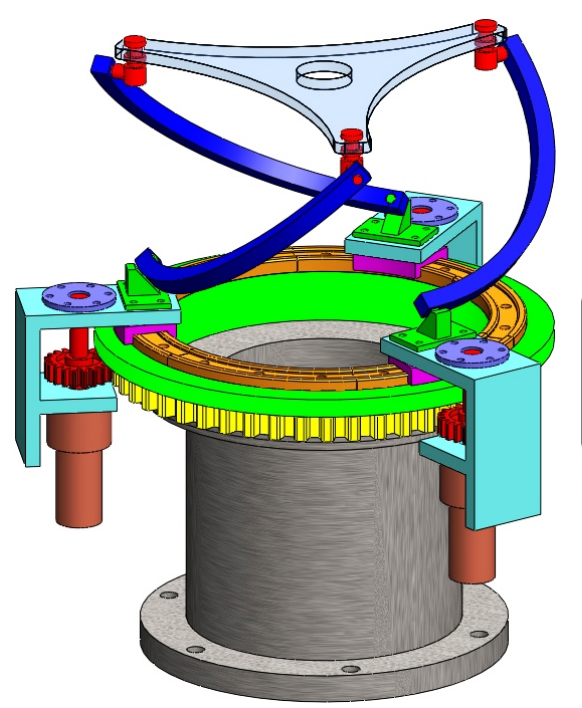

(a)

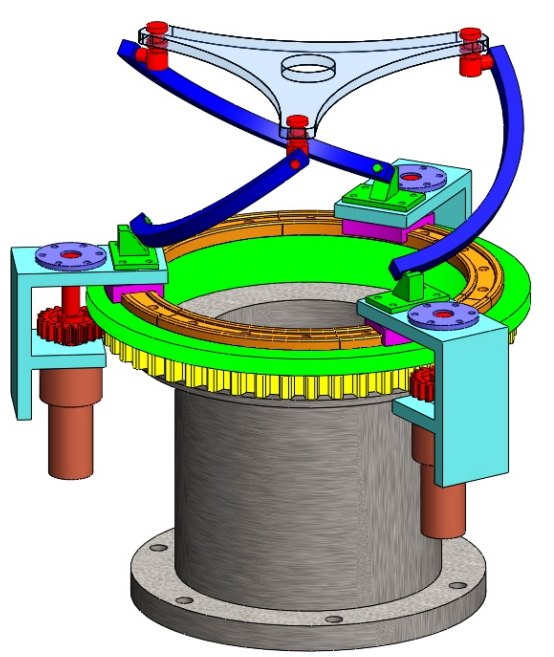

(b)

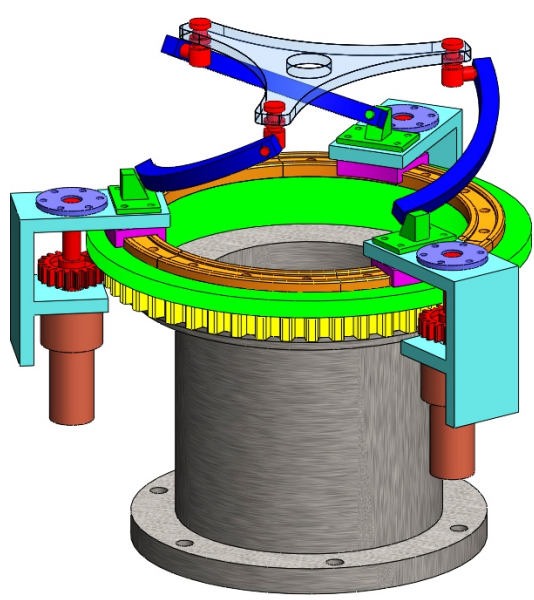

(c)

Figure 9: CAD designs of three Pareto-optimal solutions: (a) ID-I, (b) ID-II, (c) ID-III. 


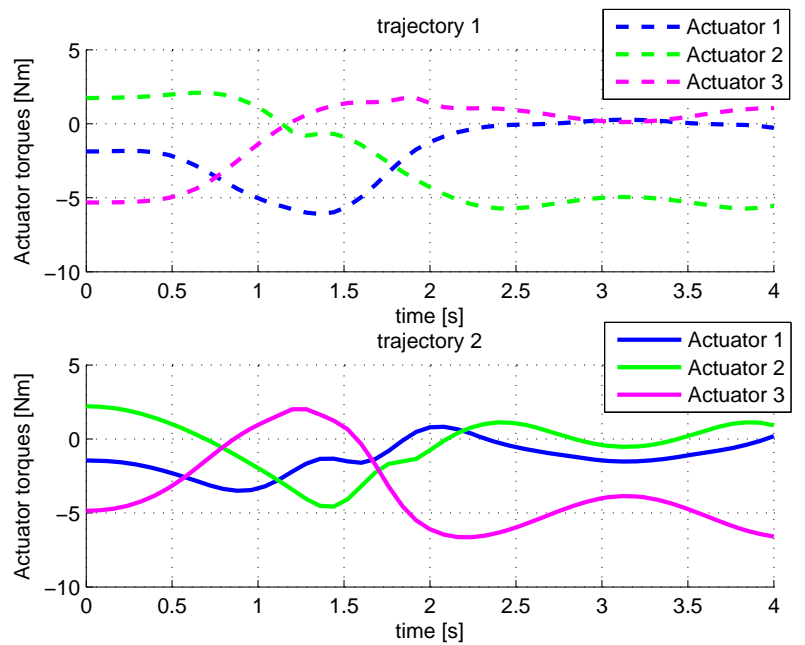

(a)

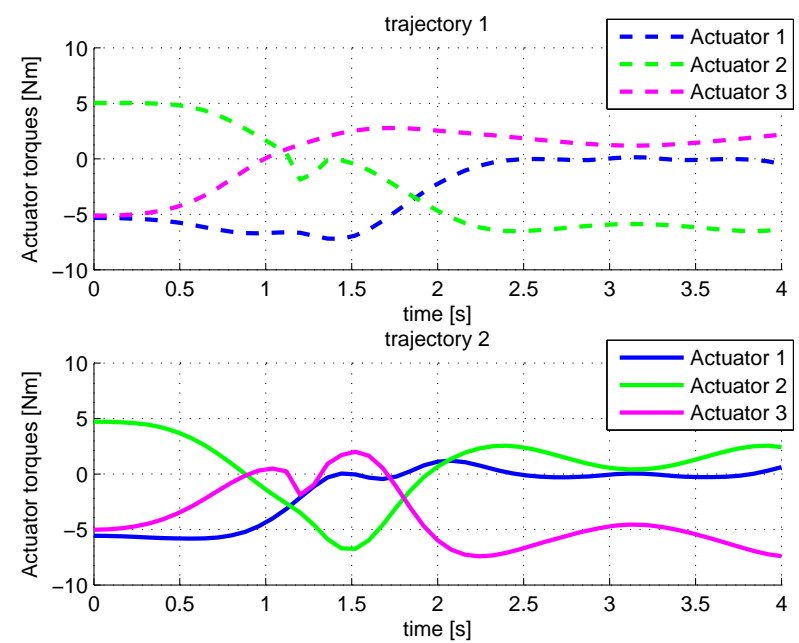

(b)

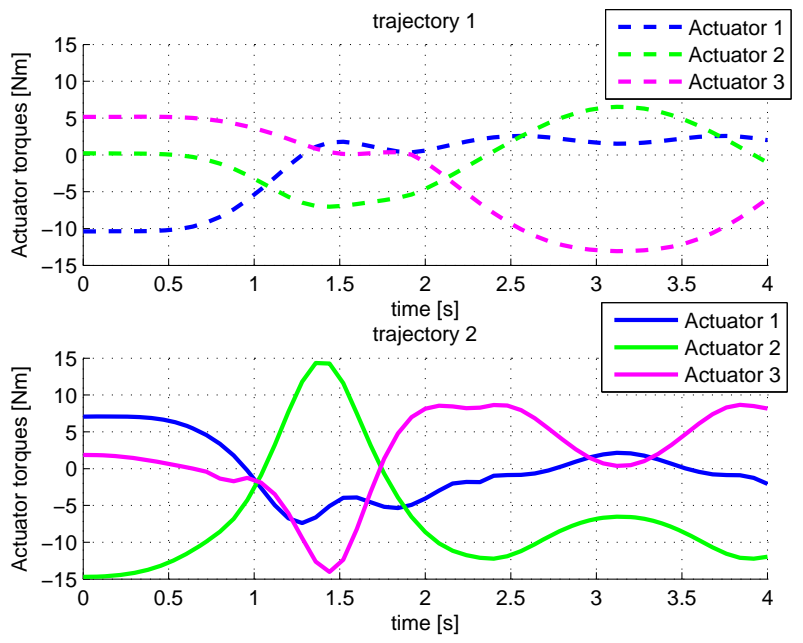

(c)

Figure 10: The dynamic simulation results for the three Pareto-optimal solutions: (a) ID-I; (b) ID-II; (c) ID-III. 


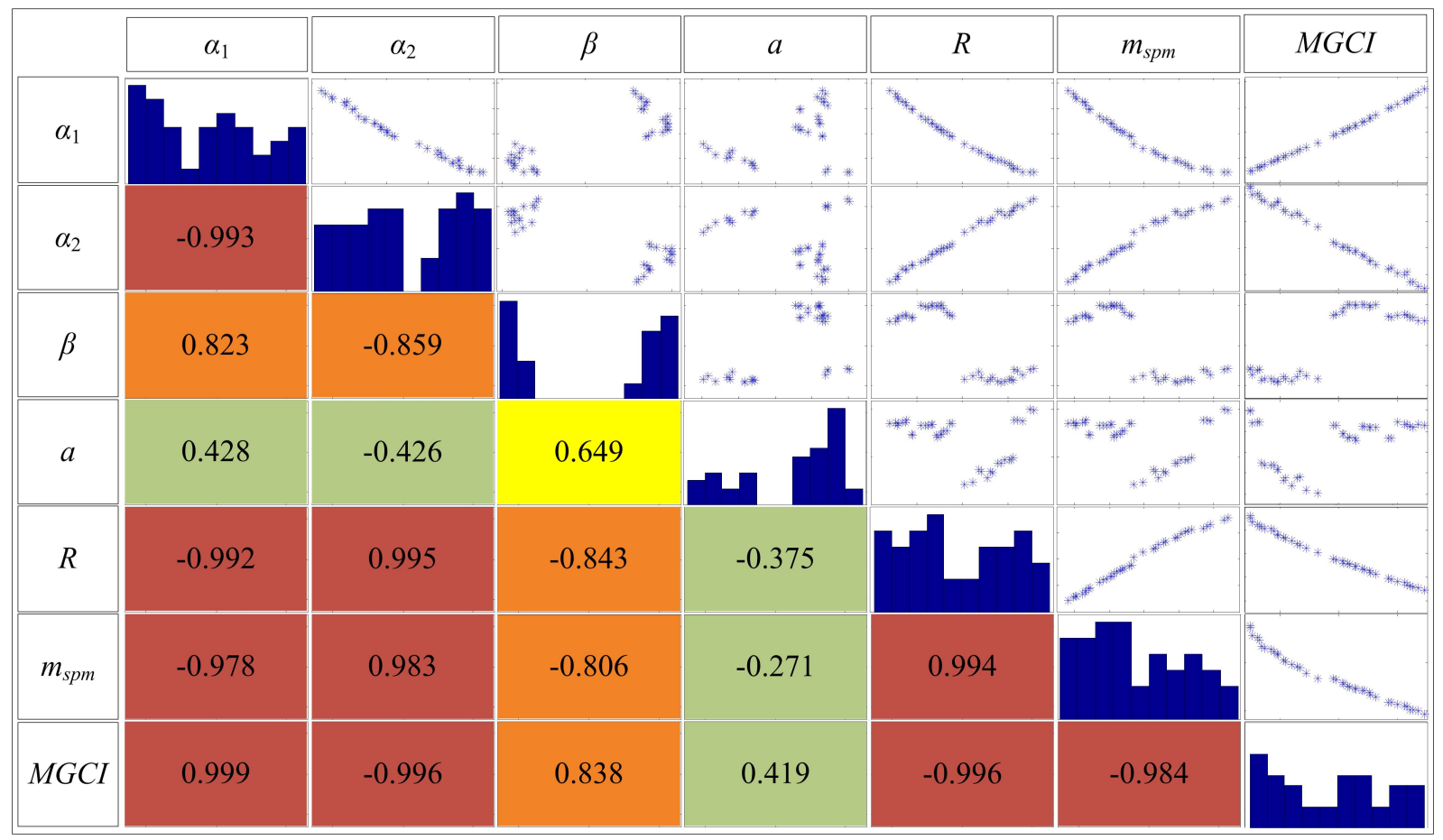

Figure 11: Scatter matrix for the objective functions and the design variables.

trends as well as the inter-dependency between the objective functions and design variables by means of a scatter matrix $[11,18]$. The lower triangular part of the matrix represents the correlation coefficients whereas the upper one shows the corresponding scatter plots. The diagonal elements represent the probability density charts of each variable. The correlation coefficients vary from -1 to 1 . Two variables are strongly dependent when their correlation coefficient is close to -1 or 1 and independent when the latter is null. Figure 11 shows that:

- $m_{\text {spm }}$ and $M G C I$ are strongly dependent as their correlation coefficient is equal to -0.984 ;

- $m_{\text {spm }}$ and $M G C I$ are strongly dependent of the design variables except $a$ as the mass of the sliding units mainly affects the total mass, thus, $a$ slightly affects $m_{\text {spm }}$;

- Both objective functions are approximately linearly related to variables $\alpha_{1}, \alpha_{2}$ and $R$;

- All the variables are strongly dependent of each other except $a$;

- The results show that $\beta$ is close to $90^{\circ}$ for all Pareto-optimal solutions;

- It is noteworthy that the higher $m_{\text {spm }}$, the lower $\alpha_{1}$. Conversely, the higher $m_{\text {spm }}$, the higher $\alpha_{2}$. Higher $R$ results in higher $m_{\text {spm }}$. 


\section{Conclusions}

In this paper, the inverse dynamics and geometric synthesis of spherical parallel manipulators were discussed. Using the classical method of Lagrange multipliers, the equations of motion for the SPMs were derived. The expressions for the kinetic energy are associated with the characteristics of motion, namely, all the SPM bodies rotating about the center of rotation. All the moving bodies are taken into account to describe this dynamic system effectively and clearly. The developed dynamic model is integrated into the design optimization procedure of the SPMs.

A multiobjective design optimization problem was formulated in order to determine the mechanism optimum structural and geometric parameters. The objective functions were evaluated based on the kinematic and kinetostatic/dynamic performances of the manipulators. This approach has been illustrated with the optimum design of an unlimited-roll spherical parallel manipulator, aiming at minimizing the mechanism mass and increasing its dexterity. As a result, the Pareto-front was obtained to show the approximation of the optimal solutions between the various (antagonistic) criteria, subject to the dependency of the performance. It turns out that the manipulator has the best performance with $\beta=90^{\circ}$.

As a matter of fact, the method offers a great flexibility to select any criterion as an objective function based on requirements. A contribution of the work is the formulation of different kinds of performances ranging from kinematics, statics to dynamics. All these formulations ease the modeling and simulation, and can be used for other design optimization tasks in future works.

\section{References}

[1] C.M. Gosselin, J. Angeles, The optimum kinematic design of a spherical three-degree-of-freedom parallel manipulator, ASME J. Mechanisms, Transmissions, and Automation in Design 111 (1989) 202-207.

[2] F. Bulca, J. Angeles, P.J. Zsombor-Murray, On the workspace determination of spherical serial and platform mechanisms, Mechanism and Machine Theory 34 (3) (1999) 497-512.

[3] S. Bai, Optimum design of spherical parallel manipulators for a prescribed workspace, Mechanism and Machine Theory 45 (2) (2010) 200-211.

[4] C.M. Gosselin, J. Angeles, A global performance index for the kinematic optimization of robotic manipulators, ASME J. Mechanical Design 113 (3) (1991) 220-226.

[5] C.M. Gosselin, E. Lavoie, On the kinematic design of spherical three-degree-of-freedom parallel manipulators, The International Journal of Robotics Research 12 (4) (1993) 394-402.

[6] S. Bai, M.R. Hansen, T.O. Andersen, Modelling of a special class of spherical parallel manipulators with Euler parameters, Robotica 27 (2) (2009) 161-170. 
[7] S. Staicu, Recursive modelling in dynamics of Agile Wrist spherical parallel robot, Robotics and Computer-Integrated Manufacturing 25 (2) (2009) 409-416.

[8] I.A. Bonev, C.M. Gosselin, Singularity Loci of Spherical Parallel Mechanisms, IEEE International Conference on Robotics and Automation, Barcelona, Spain, 2005, pp.2957-2962.

[9] X.J. Liu, Z.L. Jin, F. Gao, Optimum design of 3-DOF spherical parallel manipulators with respect to the conditioning and stiffness indices, Mechanism and Machine Theory 35 (9) (2000) $1257-1267$.

[10] F. Bidault, C.-P. Teng, J. Angeles, Structural optimization of a spherical parallel manipulator using a two-level approach, Proc. ASME 2001 Design Engineering Technical Conferences, Pittsburgh, Pennsylvania, 2001, pp.9-12.

[11] S. Caro, D. Chablat, R. Ur-Rehman, P. Wenger, Multiobjective design optimization of 3-PRR planar parallel manipulators, Global Product Development (2011) 373-383.

[12] C.M. Gosselin, J.F. Hamel, The Agile Eye: a high-performance three-degree-of-freedom cameraorienting device, IEEE International Conference on Robotics and Automation, San Diego, CA, 1994, pp.781-786.

[13] T. Li, S. Payandeh, Design of spherical parallel mechanisms for application to laparoscopic surgery, Robotica 20 (2) (2002) 133-138.

[14] H. Asada, J. Granito, Kinematic and static characterization of wrist joints and their optimal design, IEEE International Conference on Robotics and Automation, 1985, pp.244-250.

[15] M. Ceccarelli, G. Carbone, E. Ottaviano, Multi criteria optimum design of manipulators, Bulletin of the Polish Academy of Technical Sciences 53 (1) (2005) 9-18.

[16] O. Altuzarra, O. Salgado, A. Hernandez, J. Angeles, Multiobjective optimum design of a symmetric parallel schönflies-motion generator, ASME J. Mechanical Design 131 (3) (2009) 031002.

[17] D. Chablat, S. Caro, R. Ur-Rehman, P. Wenger, Comparison of planar parallel manipulator architectures based on a multi-objective design optimization approach, ASME 2010 International Design Engineering Technical Conferences and Computers and Information in Engineering Conference, Montreal, Quebec, Canada, 2010, pp.861-870.

[18] R. Ur-Rehman, S. Caro, D. Chablat, P. Wenger, Multi-objective path placement optimization of parallel kinematics machines based on energy consumption, shaking forces and maximum actuator torques: Application to the Orthoglide, Mechanism and Machine Theory 45 (8) (2010) 1125-1141.

[19] I.A. Bonev, Direct kinematics of zero-torsion parallel mechanisms, IEEE International Conference on Robotics and Automation, Pasadena, California, USA, 2008, pp.3851-3856. 
[20] J. Diebel, Representing attitude: Euler angles, unit quaternions, and rotation vectors, Stanford University Technical Report, Stanford University, Stanford, CA, 2006.

[21] J. G. Jalón, E. Bayo, Kinematic and dynamic simulation of multibody eystems: The real-Time challenge, Springer-Verlag, New-York, 1994.

[22] L. Zhou, S. Bai, M.R. Hansen, Integrated dimensional and drive-train design optimization of a light-weight anthropomorphic arm, Robotics and Autonomous Systems 60 (1) (2012) 113-122.

[23] Maxon products catalog (2012), http://www.maxonmotor.com/maxon/view/catalog/.

[24] K. Deb, A. Pratap, S. Agarwal, T. Meyarivan, A fast and elitist multiobjective genetic algorithm: NSGA-II, IEEE Trans. Evolutionary Computation 6 (2) (2002) 182-197.

[25] J. Awrejcewicz, Z. Koruba, Equations of Motion of a Rigid Spherical Body, Advances in Mechanics and Mathematics: Classical Mechanics 30 (2012) 87-123.

[26] A. Pashkevich, D. Chablat, P. Wenger, Stiffness analysis of overconstrained parallel manipulators, Mechanism and Machine Theory 44 (5) (2009) 966-982.

[27] G. Wu, S. Bai, J. Kepler, Mobile platform center shift in spherical parallel manipulators with flexible limbs, Mechanism and Machine Theory 75 (2014) 12-26.

\section{Appendix A: Mass Moment of Inertia}

The mass moment of inertia of the mobile platform about point $O$ [25] is given by

$$
\mathbf{I}_{p}=m_{p} R^{2} \cos ^{2} \beta[\mathbf{p}]_{\times}[\mathbf{p}]_{\times}^{T}+\mathbf{Q} \mathbf{I}_{p}^{\prime} \mathbf{Q}^{T}
$$

where $m_{p}$ is the mass, $\mathbf{I}_{p}^{\prime}$ is the inertia tensor in its local frame $x_{p} y_{p} z_{p}$, and $[\mathbf{p}]_{\times}=\operatorname{CPM}(\mathbf{p})$ is the skew-symmetric matrix, $\mathbf{p}$ being the unit vector of $z_{p}$ axis in Fig. 4.

A parameterized curved link with uniform cross-section is shown in Fig. 12, its center of mass being found as

$$
R \alpha \bar{x}=R^{2} \int_{-\frac{\alpha}{2}}^{\frac{\alpha}{2}} \cos \varphi \mathrm{d} \varphi \quad \text { or } \quad \bar{x}=\frac{2 R}{\alpha} \sin \frac{\alpha}{2}
$$

Let the link's mass be $m_{l}$, its moment of inertia about point $O$ is found as [25]:

$$
\mathbf{I}_{l}=m_{l} \bar{x}^{2}[\mathbf{i}]_{\times}[\mathbf{i}]_{\times}^{T}+\mathbf{E I}_{l}^{\prime} \mathbf{E}^{T}
$$

where $[\mathbf{i}]_{\times}=\operatorname{CPM}(\mathbf{i})$, and $\mathbf{E}=[\mathbf{i} \mathbf{j} \mathbf{k}], \mathbf{i}, \mathbf{j}, \mathbf{k}$ being the unit vectors of $x$-, $y$ - and $z$-axes. Moreover, 


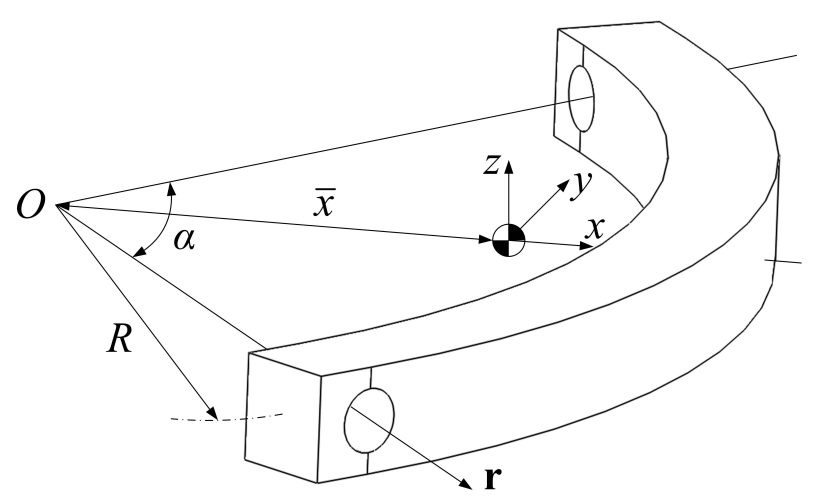

Figure 12: A curved link.

the mass moments of inertia $\mathbf{I}_{l}^{\prime}=\operatorname{diag}\left[L_{x x} L_{y y} L_{z z}\right]$ in the frame $(x, y, z)$ are expressed as:

$$
\begin{aligned}
& L_{x x}=\int_{-\frac{\alpha}{2}}^{\frac{\alpha}{2}}(R \sin \varphi)^{2} \cdot \frac{m_{l}}{R \alpha} \cdot R \mathrm{~d} \varphi=\frac{1}{2} m_{l} R^{2}\left(1-\frac{\sin \alpha}{\alpha}\right) \\
& L_{y y}=\int_{-\frac{\alpha}{2}}^{\frac{\alpha}{2}}(R \sin \varphi-\bar{x})^{2} \cdot \frac{m_{l}}{R \alpha} \cdot R \mathrm{~d} \varphi=\frac{1}{2} m_{l}\left(\frac{R^{2} \sin \alpha}{\alpha}-\frac{8 R \bar{x}}{\alpha} \sin \frac{\alpha}{2}+R^{2}+2 \bar{x}^{2}\right) \\
& L_{z z}=\int_{-\frac{\alpha}{2}}^{\frac{\alpha}{2}}\left[(R \sin \varphi)^{2}+(R \cos \varphi-\bar{x})^{2}\right] \cdot \frac{m_{l}}{R \alpha} \cdot R \mathrm{~d} \varphi=\frac{1}{2} m_{l}\left(R^{2}+\bar{x}^{2}-\frac{4 R \bar{x}}{\alpha} \sin \frac{\alpha}{2}\right)
\end{aligned}
$$

Moreover, the mass moment of inertia about $\mathbf{r}$ is derived as

$$
I_{l}=m_{l}\left(\bar{x} \sin \frac{\alpha}{2}\right)^{2}+\int_{0}^{\alpha}(R \sin \varphi)^{2} \cdot \frac{m_{l}}{R \alpha} \cdot R \mathrm{~d} \varphi=\frac{1}{4} m_{l} R^{2}\left[\frac{(1-\cos \alpha)^{2}}{\alpha^{2}}+2-\frac{\sin 2 \alpha}{\alpha}\right]
$$

\section{Appendix B: Cartesian stiffness matrix}

With the virtual-spring approach [26], the Cartesian stiffness matrix $\mathbf{K}$ of the SPM is found as

$$
\mathbf{K}=\sum_{i=1}^{3} \mathbf{K}_{i}
$$

Here $\mathbf{K}_{i} \in \mathbb{R}^{6}$ is the Cartesian stiffness matrix of the $i$ th leg, extracted from the first six-dimensional block of matrix $\mathbf{K}_{i}^{\prime}$ as below

$$
\mathbf{K}_{i}^{\prime}=\left[\begin{array}{cc}
\mathbf{J}_{\theta}^{i}\left(\mathbf{K}_{\theta}^{i}\right)^{-1} \mathbf{J}_{\theta}^{i^{T}} & \mathbf{J}_{q}^{i} \\
\mathbf{J}_{q}^{i T} & \mathbf{0}_{2}
\end{array}\right]^{-1}
$$




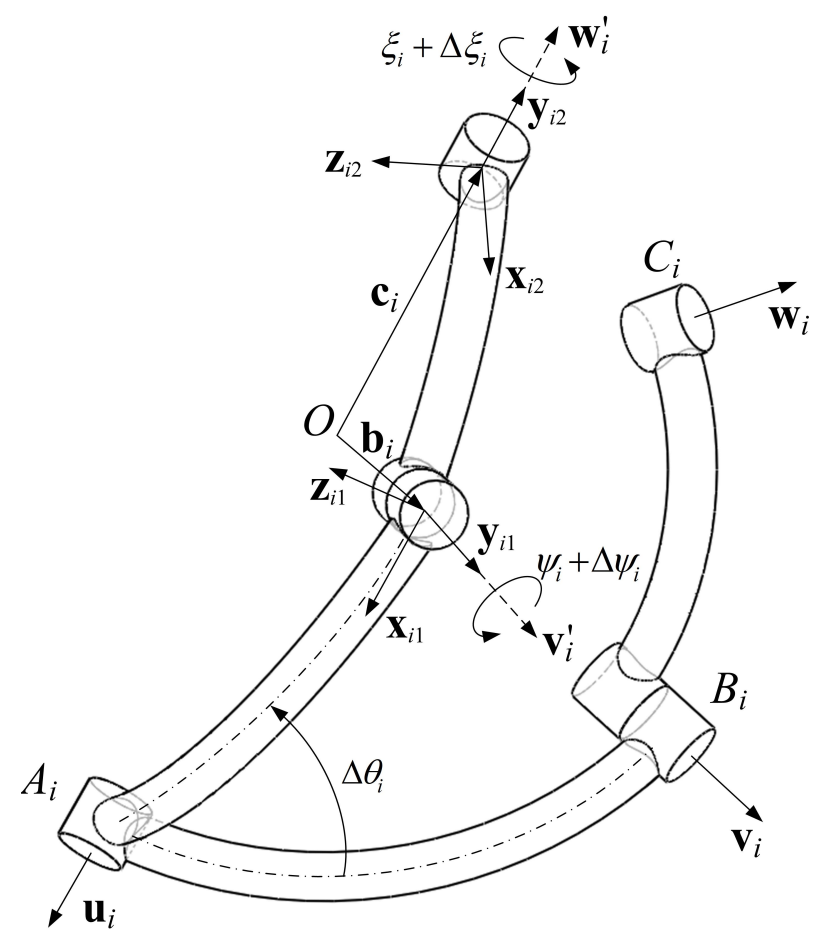

Figure 13: Link deflections and joint displacements of a flexible leg.

with

$$
\begin{aligned}
\mathbf{J}_{\theta}^{i} & =\left[\begin{array}{llll}
\hat{\boldsymbol{\$}}_{A}^{i} & \hat{\boldsymbol{\$}}_{u 1}^{i} & \ldots & \hat{\boldsymbol{\$}}_{u 12}^{i}
\end{array}\right] \in \mathbb{R}^{6 \times 13} \\
\mathbf{J}_{q}^{i} & =\left[\begin{array}{ll}
\hat{\boldsymbol{\$}}_{B}^{i} & \hat{\boldsymbol{\$}}_{C}^{i}
\end{array}\right] \in \mathbb{R}^{6 \times 2}
\end{aligned}
$$

According to Fig. 13, the unit screws are given by

$$
\begin{aligned}
& \hat{\boldsymbol{\$}}_{A}^{i}=\left[\begin{array}{c}
\mathbf{u}_{i} \\
\mathbf{0}
\end{array}\right], \hat{\boldsymbol{\$}}_{B}^{i}=\left[\begin{array}{c}
\mathbf{v}_{i} \\
\mathbf{0}
\end{array}\right], \hat{\boldsymbol{\$}}_{C}^{i}=\left[\begin{array}{c}
\mathbf{w}_{i} \\
\mathbf{0}
\end{array}\right] \\
& \hat{\boldsymbol{\$}}_{u 1}^{i}=\left[\begin{array}{c}
\mathbf{x}_{i 1} \\
\mathbf{b}_{i} \times \mathbf{x}_{i 1}
\end{array}\right], \hat{\boldsymbol{\$}}_{u 2}^{i}=\hat{\boldsymbol{\$}}_{B}^{i}, \hat{\boldsymbol{\$}}_{u 3}^{i}=\left[\begin{array}{c}
\mathbf{z}_{i 1} \\
\mathbf{b}_{i} \times \mathbf{z}_{i 1}
\end{array}\right], \hat{\boldsymbol{\$}}_{u 4}^{i}=\left[\begin{array}{c}
\mathbf{0} \\
\mathbf{x}_{i 1}
\end{array}\right], \hat{\boldsymbol{\$}}_{u 5}^{i}=\left[\begin{array}{c}
\mathbf{0} \\
\mathbf{v}_{i}
\end{array}\right], \hat{\boldsymbol{\$}}_{u 6}^{i}=\left[\begin{array}{c}
\mathbf{0} \\
\mathbf{z}_{i 1}
\end{array}\right] \\
& \hat{\boldsymbol{\$}}_{u 7}^{i}=\left[\begin{array}{c}
\mathbf{x}_{i 2} \\
\mathbf{c}_{i} \times \mathbf{x}_{i 2}
\end{array}\right], \hat{\boldsymbol{\$}}_{u 8}^{i}=\hat{\boldsymbol{\$}}_{C}^{i}, \hat{\boldsymbol{\$}}_{u 9}^{i}=\left[\begin{array}{c}
\mathbf{z}_{i 2} \\
\mathbf{c}_{i} \times \mathbf{z}_{i 2}
\end{array}\right], \hat{\boldsymbol{\$}}_{u 10}^{i}=\left[\begin{array}{c}
\mathbf{0} \\
\mathbf{x}_{i 2}
\end{array}\right], \hat{\boldsymbol{\$}}_{u 11}^{i}=\left[\begin{array}{c}
\mathbf{0} \\
\mathbf{w}_{i}
\end{array}\right], \hat{\boldsymbol{\$}}_{u 12}^{i}=\left[\begin{array}{c}
\mathbf{0} \\
\mathbf{z}_{i 2}
\end{array}\right]
\end{aligned}
$$

and $\mathbf{K}_{\theta}^{i} \in \mathbb{R}^{13}$ describes the stiffness of the actuation and virtual springs, taking the form:

$$
\mathbf{K}_{\theta}^{i}=\operatorname{diag}\left[\begin{array}{lll}
K_{a c t}^{i} & \mathbf{K}_{L_{1}}^{i} & \mathbf{K}_{L_{2}}^{i}
\end{array}\right]
$$

where $K_{a c t}^{i}$ is the $i$ th actuator stiffness, $\mathbf{K}_{L_{1}}^{i}$ and $\mathbf{K}_{L_{2}}^{i}$, respectively, are the $6 \times 6$ stiffness matrices of the proximal and distal curved links in the $i$ th leg, which can be found in [27]. 